\title{
LIST OF ABBREVIATIONS USED FOR LABOR ORGANIZATIONS AND POLITICAL PARTIES
}

CBEU, Ceylon Bank Employees' Union

CCCTU, Central Council of Ceylon Trade Unions

CESU, Ceylon Estates Staff' Union

CFL, Ceylon Federation of Labour

CFTU, Ceylon Federation of Trade Unions

CMU, Ceylon Mercantile Union

CPWU, Ceylon Plantation Workers' Union

CTUF, Ceylon Trade Union Federation

CWC, Ceylon Workers' Congress

DWC, Democratic Workers' Congress

GCSU, Government Clerical Service Union

GWTUF, Government Workers' Trade Union Federation

JCTUO, Joint Committee of Trade Union Organisations

LEWU, 'Lanka Estate Workers' Union

LSSP, Lanka Sama Samaja Party

LSSP(R), Lanka Sama Samaja Party (Revolutionary)

MEP, Mahajana Eksath Peramuna

PSWTUF, Public Service Workers' Trade Union Federation

SLFP, Sri Lanka Freedom Party

SLJGS, Sri Lanka Jathika Guru Sangamaya

SLNVSS, Sri Lanka Nidahas Vurthiya Samithi Sammelanaya

SLRLS, Samastha Lanka Rajaye Lipikaru Sangamaya

UCCTU, United Committee of Ceylon Trade Unions

UNP, United National Party

UPTO, Union of Post and Telecommunication Officers

UPWU, United Port Workers' Union

VLSSP, Viplavakari Lanka Sama Samaja Party 
